\title{
Hexagonal CoOOH Nanoflakes Synthesized From Three-Dimensional Cellulose Membrane Matrix With Enhanced Detection of Ascorbic Acid
}

Jian Xia

Huazhong University of Science and Technology

Mengru Yang

Wuhan Institute of Technology

Xiangyang Jiang

Wuhan Institute of Technology

Mohd Shabbir

Wuhan Institute of Technology

Xiaogang Luo ( $\nabla$ xgluo0310@hotmail.com )

Wuhan Institute of Technology https://orcid.org/0000-0003-3493-2140

\section{Research Article}

Keywords: Cellulose, $\mathrm{CoOOH}$ nanoflakes, ascorbic acid, one-step method, colorimetric detection

Posted Date: June 2nd, 2021

DOI: https://doi.org/10.21203/rs.3.rs-536216/v1

License: (9) This work is licensed under a Creative Commons Attribution 4.0 International License. Read Full License 


\section{Abstract}

In this work, cobalt oxyhydroxide ( $\mathrm{CoOOH}$ ) nanoflakes/cellulose composite membranes (CCM) were fabricated by a simple one-step method and green route without further treatment. The $\mathrm{CoOOH}$ nanoflakes were synthesized in the high-purity cellulose membranes (CM) matrix that expected to enhance its detection performance of ascorbic acid in comparison to the traditional paper-based sensors that were made of commercially available filter paper. The structure and properties of CM and CCM were characterized by SEM, EDS, FT-IR, XRD and XPS, etc. The combination mechanism between nanoflakes and cellulose was well analyzed and discussed by various characterizations and experiments. When the CCM was immersed into the ascorbic acid (AA) solution, the color changing from colorless to blue was observed only in 2 min. Furthermore, the CCM can be used for AA determination in real samples.

\section{Introduction}

Ascorbic acid (AA) is an essential micronutrient for human body and abnormal levels of AA lead to many diseases, such as the common cold, scurvy, mental illness, atherosclerosis, cancers, and cataracts. (Esteban and Pei 2012; Liu et al. 2013) It is crucial to understand the biological and pathological roles of AA in brain system where micromolar levels are typical much lower than other humoral fluids. Many tools, such as liquid chromatography (LC) assay, fluorescence sensors,(Xie et al. 2020) electrochemistry sensors(Liu et al. 2013) and so on, have been developed for the detection of ascorbic acid, which exhibited higher response to AA detection. Nevertheless, most of them require complex processes and expensive equipment, limiting its application for real-time and direct point of care detection. Therefore, it is a current demand to develop a simple, low-cost and fast platform for AA detection without any complicated auxiliary tools.

Recently, there are a lot of nanomaterials have been developed for AA detection.(Han et al. 2019; Tokura et al. 2019) However, the fabrication method of most nanomaterials in these reported works were inevitably faced complicated, toxic, and time-consuming routes. Compared to these methods, $\mathrm{CoOOH}$ nanoflakes can be used to solve this problem well, and it also has been intensively explored for AA detection.(Fang et al. 2019; Ji et al. 2018; Nie et al. 2018) As an amphoteric hydroxide, $\mathrm{CoOOH}$ nanoflakes can be easily synthesized by a simple redox method.(Ji et al. 2018) Nevertheless, CoOOH nanoflakes synthesized by redox method have a poor colorimetric effect for AA detection because the inherent high surface energy of nanoflakes can easily lead to the aggregation of $\mathrm{CoOOH}$ nanoflakes. In addition, in these reported works about $\mathrm{CoOOH}$ nanoflakes, their fabrication method was mainly through simple redox reaction and ultrasonic treatment, not conducing to the size control of $\mathrm{CoOOH}$ nanoflakes and longterm storage. Therefore, it is very necessary to find a suitable carrier to improve the dispersibility and stability of the $\mathrm{CoOOH}$ nanoflakes, thereby enhancing the detection ability of nanoflakes to AA.

Recent years evidence about cellulose-based materials' wide utilization in different application areas such as low-cost diagnostics,(Guadarrama-Fernandez et al. 2018; Mahato et al. 2017; Tseng et al. 2018) hincancare (Cantnc ot al 2018) and drun narrier(Luo et al. 2016b). However, the substrates of most paperLoading [MathJax]/jax/output/CommonHTML/jax.js 
based test strips are made of filter paper, limiting its further functional application because of their uncontrollability of composition and morphology structure. In our previous work, a simple cellulose-based strips based on high-purity cellulose membrane was developed and achieved a good detection result compared with traditional paper-based sensors.(Luo et al. 2019) Therefore, the use of high-purity cellulose membrane prepared via a simple and green route to design the nanflakes/cellulose hybrid membrane can be expected to effectively improve the detection ability of hybrid membrane.

In this work, $\mathrm{CoOOH}$ nanoflakes were synthesized from water-soluble cellulose solution by a simple onestep method. In the hybrid systems, $\mathrm{NaOH}$ not only promoted the dissolution of cellulose, but also acted as a participating reagent to promote the formation of $\mathrm{CoOOH}$ nanoflakes. In addition, the interpenetrating porous network structure of regenerated cellulose matrix is conducive to the dispersion of nanoflakes and can be used as a micro reactor to control the growth of nanoflakes.(Van Rie and Thielemans 2017) The combination between nanoflakes and cellulose makes the hybrid membrane expected to be an excellent detector for AA detection. The coagulation mechanism of $\mathrm{CoOOH}$ nanoflakes/cellulose composite membrane was also deeply studied, and its detection performance for AA was well evaluated. This work has provided an appropriate strategy for the combination of threedimensional inorganic materials and natural polymer materials, which is hopeful to expand the applications of natural polymer-based functional materials in disease diagnosis, food safety and cosmetic.

\section{Experimental Section}

\subsection{Materials}

Cellulose (Cotton linter pulp; \-cellulose > 95\%) was provided by Hubei Chemical Fiber Group Ltd. (Xiangfan, China). Its viscosity-average molecular weight in cadoxen was determined using an Ubbelohde viscometer at $25^{\circ} \mathrm{C}$ to be $9.6 \times 10^{4}$ according to Mark - Houwink equation $[\mathrm{n}](\mathrm{mL} / \mathrm{g})=3.85 \times 10-$ $12\left(\mathrm{M}_{\mathrm{w}}\right)^{0.76}$.(Ye et al. 2017) Sodium hypochlorite $(\mathrm{NaClO})$, ascorbic acid ( $\left.\mathrm{AA}\right)$, sodium hydroxide $(\mathrm{NaOH})$ and cobaltous chloride hexahydrate $\left(\mathrm{CoCl}_{2} \cdot 6\left(\mathrm{H}_{2} \mathrm{O}\right)\right)$ were purchased from Sinopharm Chemical Reagent Co., Ltd. (Shanghai, China). 3,3',5,5'-tetramethylbenzidine (TMB), lactose, maltose, sucrose, fructose, and glucose were purchased from Sigma-Aldrich. Water used in all experiments was distilled water purified by the Ultra-pure Water System (Heal Force, Shanghai, China). Other chemicals of analytical grade were supplied by the Sinopharm Chemical Reagent Co., Ltd (Shanghai, China).

\subsection{Preparation of $\mathrm{CoOOH}$ nanoflakes/cellulose composite membranes}

The $\mathrm{CoOOH}$ nanoflakes/cellulose composite membranes (CCM) were fabricated by a simple one-step method based on our previous works with minor modification.(Xia et al. 2020a) Typically, cotton linter was dissolved into $\mathrm{NaOH} / \mathrm{urea} / \mathrm{H}_{2} \mathrm{O}$ system to obtain the clear cellulose solution, and then $1.275 \mathrm{~mL}$ 
stirring for $5 \mathrm{~min}$. The mixture solution was used to prepare the CCM by a simple casting method after centrifugation. Finally, the CCM was rinsed with deionized water for 3 times and lyophilized. In addition, pure $\mathrm{CoOOH}$ nanoflakes and cellulose membrane were also fabricated for further characterization according to the previous reported work, respectively.(Ji et al. 2018; Xia et al. 2020b) The detailed preparation information was given in the Supporting Information.

\subsection{Characterizations}

The surface morphology and structure of $\mathrm{CoOOH}$ nanoflakes, $\mathrm{CM}$ and $\mathrm{CCM}$ were investigated by scanning electron microscope (SEM; JSW-5510LV) at a voltage of $2.0 \mathrm{kV}$, and the elemental concentration was measured by means of Energy Dispersive X-ray mappings (EDS; FALCON60). For other characterizations, the samples were cut into powders and dried in vacuum oven over at $60^{\circ} \mathrm{C}$ for $48 \mathrm{~h}$. Dry powder samples were pretreated with potassium bromide pellet pressing method and then were used for the FT-IR tests. Fourier transform infrared (FT-IR) spectra were taken on a FT-IR spectrometer (NICOLET 6700 ) and the number of FT-IR scans was 64 times with a range of $4000-500 \mathrm{~cm}^{-1}$. The crystal structure property of powder samples was customized by X-ray diffraction (XRD; D8 ADVANCE) with a Cu tube wavelength of $1.5418 \mathrm{~A}^{\circ}$. Scans were taken over a $2 \theta$ range from 10 to $80^{\circ}$ with a step of $0.02^{\circ}$ using a $10 \%$ min scanning speed at a voltage of $40 \mathrm{kV}$ and a current of $40 \mathrm{~mA}$. The existence of atoms on the surface of the composite nanofibrous mats was analyzed by X-ray photoelectron spectroscopy (XPS, ESCALAB Xi+) with Al K Alpha at an energy step size of $0.05 \mathrm{eV}$.

\subsection{Colorimetric detection of ascorbic acid}

The procedures of AA detection were carried out as follows: firstly, CCM was cut into several small strips $(5 \mathrm{~mm} \cdot 20 \mathrm{~mm})$ and ascorbic acid solutions of various concentrations were prepared for use. Then, the CCM was immersed into the colorimetric bottle $(10 \mathrm{~mL})$ containing $4 \mathrm{~mL}$ standard solutions of $A A$ and $0.2 \mathrm{~mL}$ TMB $(30 \mathrm{mM})$. After 10 minutes of reaction, the CCM was taken out and the color images were collected with a camera, then analyzed by grey scale intensity with Photoshop software.(Apilux et al. 2012) The composited membranes after reacted with AA solution were named as CCM-AA.

\subsection{Detection of AA in real samples}

The orange (fruit sample) was chosen as the real sample for AA detection. Orange was purchased from the local supermarket in Wuhan. Collected orange samples were rinsed with deionized water and orange peel was removed. Then, the orange meat was chopped and pressed into juice that was further subjected to decolorizing by activated charcoal. Each sample was spiked with various concentrations of ascorbic acid. After that, the real samples were detected by using the CCM and the color images were collected with a camera. A control test was carried out by using the CCM immersed into the blank solution.(Su et al. 2012)

\section{Results And Discussion}


The morphological structure of $\mathrm{CM}, \mathrm{CCM}$ and $\mathrm{CoOOH}$ nanoflakes were investigated by SEM (Fig. 1). CM (Fig. 1a) exhibited a loose and porous network structure, which was conducive to the dispersion and size control of $\mathrm{CoOOH}$ nanoflakes in $\mathrm{CM}$. Porous network structure of $\mathrm{CM}$ was believed to be formed by reaggregation of cellulose molecular chains during the regeneration of cellulose, forming a dense hydrogen bonding network. Figure $1 \mathrm{~b}$ shows the surface morphology of $\mathrm{CCM}$, and the porous network structure was still preserved in $\mathrm{CCM}$, but most of them were covered by $\mathrm{CoOOH}$. The morphology of pure $\mathrm{CoOOH}$ nanoflakes is shown in Fig. 1c. They were obtained in hexagonal shapes with a size distribution of about $100 \mathrm{~nm}$, consequently provide a large surface area to react with TMB.(Ji et al. 2018) Compared with pure $\mathrm{CoOOH}$ nanoflakes, the polygonal nanoflakes existed in CCM indicated that the formation of $\mathrm{CoOOH}$ nanoflakes in cellulose matrix. The element type and content of the surface of $\mathrm{CoOOH}$ nanoflakes were analyzed by EDS patterns (Fig. 1d). Peaks from Co and $O$ were observed in EDS patterns and the atom ratio of $\mathrm{Co}$ and $\mathrm{O}$ elements in $\mathrm{CoOOH}$ nanoflakes was nearly 1:2 (Table S1), which confirmed the successful preparation of nanoflakes.(Ji et al. 2018)

The FT-IR spectra of cotton linter, $\mathrm{CoOOH}$ nanoflakes, $\mathrm{CM}, \mathrm{CCM}$ and CCM-AA are shown in Fig. 2. There was an obvious band at about $3443 \mathrm{~cm}^{-1}$, which could be corresponding to the broad $-\mathrm{OH}$ vibration of cellulose and $\mathrm{CoOOH}$ nanoflakes (Fig. 2a-e).(Ji et al. 2018; Luo et al. 2016a) The band at $585 \mathrm{~cm}^{-1}$ (Fig. 2b) was the Co- ${ }^{2-}$ complex in the assemblies.(Ji et al. 2018; Li et al. 2019) Compared with pure cellulose membrane (Fig. 2C), the spectra of CCM and CCM-AA (Fig. $2 \mathrm{~d}$ and $2 \mathrm{e}$ ) are not show a more obvious change. The main reason is maybe that the proportion of Co element in CCM is very small so that the main characteristic band of $\mathrm{CoOOH}$ in cellulose matrix is not obvious.

The crystal structure of cotton linter, CM, CCM and CCM-AA are shown in Fig. 3. The diffraction peaks at $2 \theta=14.75^{\circ}, 16.34^{\circ}$ and $22.60^{\circ}$ in Fig. 3a were corresponding to the type I cellulose (1-10), (110) and (200) planes, while the diffraction peaks at $2 \theta=12.03^{\circ}, 20.04^{\circ}$ and $21.44^{\circ}$ in Fig. $3 \mathrm{~b}$ were corresponding

to the type Il cellulose (1 10), (110) and (020) planes, indicating the transition of cellulose crystalline form I to II.(French 2013; French 2020) Fig. 3c and 3b were the pattern of CCM and CCM-AA, there was little difference between them. The XRD pattern of $\mathrm{CoOOH}$ nanoflakes are shown in Fig. S2. The diffraction peaks at $2 \theta=20.07^{\circ}, 38.16^{\circ}$ and $50.28^{\circ}$ were corresponding to the (003), (012) and (015) planes, respectively (JCPDS: No.73-1213 and No.07-0169), it indicated that the $\mathrm{Co}^{2+}$ was converted into $\mathrm{Co}^{3+}$ by $\mathrm{ClO}^{-}$from sodium hydroxide solution.(Ding et al. 2018; Ji et al. 2018; Li et al. 2019)

To further understand the fabrication mechanism of CCM and its detection mechanism to AA, XPS spectra were used to analyze the surface elemental compositions in $\mathrm{CoOOH}$ nanoflakes, $\mathrm{CM}, \mathrm{CCM}$ and CCM-AA. Figure $4 \mathrm{a}$ and $4 \mathrm{~b}$ show the XPS survey of $\mathrm{CoOOH}$ nanoflakes, CM, CCM and CCM-AA, the peak at $779.9 \mathrm{eV}$ confirmed the existence of Co element in CCM. Figure 4c shows the Co $2 p$ spectrum of $\mathrm{CoOOH}$ nanoflakes and CCM, the two peaks at $780.6 \mathrm{eV}$ and $795.6 \mathrm{eV}$ are corresponding to $\mathrm{Co}_{3 / 2}$ and $\mathrm{Co}_{1 / 2}$, respectively, which confirmed the formation of $\mathrm{CoOOH}$ nanoflakes in cellulose membrane.(Li et al. 2014; Li et al. 2019) Fig. 4d shows the 01 s spectrum of $\mathrm{CoOOH}$ nanoflakes, CM, CCM and CCM-AA. 
the reason could be that some Co-O bonds introduced into the CCM. Figure $4 \mathrm{e}$ and $4 \mathrm{f}$ show the $01 \mathrm{~s}$ spectrum of $\mathrm{CoOOH}$ nanoflakes and CCM, the peak at $530.95 \mathrm{eV}$ could be assigned to $\mathrm{C}-\mathrm{O}$ bonds. (Luo et al. 2017) Fig. 4e shows the Co $2 p$ spectrum of $\mathrm{CoOOH}$ nanoflakes, the peaks at $529.3 \mathrm{eV}$ and $531.1 \mathrm{eV}$ could be assigned to $-\mathrm{O}-\mathrm{H}$ and $\mathrm{O}-\mathrm{Co}-\mathrm{O}$ in $\mathrm{CoOOH}$ nanoflakes,(Jin et al. 2019; Wang et al. 2017) respectively, while the peaks at $530.4 \mathrm{eV}, 531.3 \mathrm{eV}, 531.9 \mathrm{eV}$ and $532.5 \mathrm{eV}$ are corresponding to 0-Co-0, -0$\mathrm{H}, \mathrm{C}=\mathrm{O}$ and $\mathrm{C}-\mathrm{O}$ in $\mathrm{CCM}$ (Fig. 4f). Above results confirmed that the $\mathrm{CoOOH}$ nanoflakes were successfully synthesized in cellulose membrane.

\subsection{UV-vis spectrometer measurements of the prepared nanoflakes}

Here, pure $\mathrm{CoOOH}$ nanoflakes were prepared by a simple redox method. The optical properties of pure $\mathrm{CoOOH}$ nanoflakes was first investigated by UV-vis spectrometer. Figure 5 shows the UV-vis absorption spectra of $\mathrm{CoCl}_{2}$ and $\mathrm{CoOOH}$ nanoflakes. For $\mathrm{CoCl}_{2}$ solution, it had a maximum absorption peak at 510 $\mathrm{nm}$, while the $\mathrm{CoOOH}$ nanoflakes had a maximum absorption peak at $413 \mathrm{~nm}$.(Wen et al. 2019) The color of $\mathrm{CoCl}_{2}$ solution also changed from pink to brown during the conversion of $\mathrm{CoCl}_{2}$ to $\mathrm{CoOOH}$ nanoflakes. Above results indicated that the $\mathrm{CoOOH}$ nanoflakes could be synthesized by a simple redox method. Nevertheless, the dispersion of pure $\mathrm{CoOOH}$ nanoflakes by this method was very poor and some preliminary experiments have demonstrated that it was unable to oxidize TMB directly. Interestingly, the composite membranes exhibited strong oxidizing properties to TMB when $\mathrm{CoOOH}$ nanoflakes were synthesized in water-soluble cellulose solution (Fig. 6a).

\subsection{Colorimetric detection of ascorbic acid by using the CCM}

To study the detection ability of the prepared CCM for AA in solution, qualitative and quantitative analysis were carried out.(Wang et al. 2016) Firstly, the oxidation ability of pure $\mathrm{CoOOH}$ nanoflakes and CCM to TMB was first studied. Compared with $\mathrm{CCM}$, pure $\mathrm{COOOH}$ nanoflakes showed poor oxidation ability to TMB, while CCM exhibited good oxidation ability to TMB (Fig. S3). Then, the colorimetric detection of ascorbic acid in solution by using the CCM was studied (Fig. 6). It indicated that the CCM could oxidize the TMB into oxTMB, produced a blue color. Compared with the control group, the color intensity of CCM was getting lighter. The reason was that the $\mathrm{CoOOH}$ nanoflakes could work as the recognition unit to react with the enediol group of $A A$, resulting in the partial decomposition of nanoflakes.

Based on it, the colorimetric detection of AA with different standard concentrations by using CCM was further studied. Figure 7a shows the color development state of CCM after dipping in ascorbic acid solution. It can be concluded that the CCM can be used as a good reactor in aqueous solution and has a good color rendering effect to AA. Figure 7b shows the color change of CCM at different ascorbic acid concentrations. The color depth of CCM decreased as the AA concentrations increased, indicating that

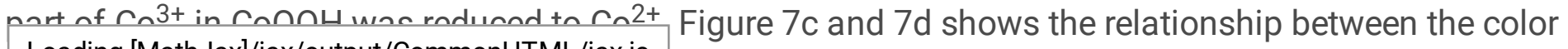
Loading [MathJax]/jax/output/CommonHTML/jax.js 
intensity on the surface of CCM and ascorbic acid concentration. It also exhibited a good linear relationship $\left(R^{2}=0.9993\right)$.

\subsection{Detection of AA in real samples}

To further verify whether the CCM could be applied to the detection of AA in real samples, several different concentrations of ascorbic acid solution were added into the real samples, where the concentration of ascorbic acid in samples 1 and 2 were 10 and $15 \mu \mathrm{M}$, respectively (Fig. 8). Compared with the blank group, the experimental results of ascorbic acid detection by colorimetric method were close to the true value within the experimental error. The detection results of different concentrations of AA solution in real samples indicated that the CCM could achieve the semi quantitative detection of AA and had a great potential to be used for disease diagnosis, food safety and cosmetic.

\subsection{Detection of other potential interferences}

To testify specific detection of ascorbic acid by CCM, the detection of other interferences was similarly studied. Sucrose, lactose, maltose, glucose, and fructose were typically chosen, and their concentrations were taken $5 \mathrm{mM}$, other conditions were the same as AA detection. Figure 9 shows the color changes of CCM upon adding those interferences. It could obviously be seen that the color intensity of AA detection was far less than the color intensity of other interferences, even though their concentration was 100 times that of AA. The main reason was that these potential interferences did not have the similar structure of enediol group, could not cause the decomposition of $\mathrm{CoOOH}$ nanoflakes. The results indicated that the CCM has a good interference resistance to other carbohydrates.

\subsection{Stability of the CCM}

The long-term stability results of the CCM are shown in Fig. 10. The samples were loaded into a ziplock bag and stored at room temperature. Figure 10 shows the color intensity changes of CCM after storing for varying number of days. Under the same detection conditions, the color intensity of CCM to AA detection was slightly decreased as the number of storing days increased. The reason may be that environmental factors cause partial decomposition of nanoflakes, or it may be a factor of light. The results indicated that the prepared CCM could be stored at room temperature for more than 60 days or even longer, suitable for long-term preservation.

\subsection{Design and detection principle of the prepared CCM}

In this work, a high-purity cellulose membrane synthesized from a water-soluble cellulose solution was used to improve $\mathrm{CoOOH}$ nanoflakes' detection efficiency to AA. The design principle of CCM for AA detection is depicted in Scheme 1. The detection mechanism of CCM for AA was that the Co-O double bond in $\mathrm{CoOOH}$ could easily dissociate by accepting electrons from TMB and convert it into oxTMB, producina a blue color. However. the color intensity of CCM would be decreased in the existence of AA Loading [MathJax]/jax/output/CommonHTML/jax.js 
solution. Based on the detection mechanism of $\mathrm{AA}$, the main problem was to immobilize the $\mathrm{CoOOH}$ nanoflakes onto the cellulose membrane. Considering that the synthesis process of $\mathrm{CoOOH}$ nanoflakes is remarkably similar to the dissolution process of cellulose, one-step method is chosen to prepare the composite membranes. More importantly, the porous network structure of cellulose membrane matrix is highly conducive to the dispersion of $\mathrm{CoOOH}$ nanoflakes. The fabrication mechanism of the composite membrane is shown in Scheme 1a, the hydroxyl groups of cellulose and the $\mathrm{CoOOH}$ nanoflakes can easily bond through the intermolecular hydrogen bonding. When the CCM immersed into the ascorbic acid solution, the color of CCM can change from colorless to blue within 2 minutes.

\section{Conclusions}

In summary, an easy, low-cost, and sensitive cellulose-based composite membranes modified with cobalt oxyhydroxide nanoflakes has been successfully developed for the low concentration AA detection. Hexagonal ultrathin $\mathrm{CoOOH}$ nanoflakes were synthesized in water-soluble cellulose solution by a simple one-step method, and the prepared CCM achieved good detection ability for AA detection. The fabrication mechanism of CCM was well characterized and confirmed by SEM, EDS, FT-IR, XRD and XPS. CoOOH nanoflakes combined with cellulose membrane matrix was mainly via the intermolecular hydrogen bonding. The porous network structure of cellulose matrix could prevent the aggregation of nanoflakes and improve its catalyst performance. Different concentrations of standard AA solutions were used to evaluate the detection performance of CCM to ascorbic acid. The results showed that CCM could achieve the rapid detection of AA within only 2 min and the color could be clearly observed by naked eyes. Besides, the practicability of CCM had also been confirmed by analysis of AA in real samples, and the color depth was recorded by a simple camera to achieve the semi quantitative detection of AA at some extent. Finally, this work provided a simple and facile design strategy for the functional design of cellulose-based membranes materials for biochemical detections.

\section{Declarations}

\section{ACKNOWLEDGMENTS}

This work was supported by the National Natural Science Foundation of China (51773159), Special Projects of the Central Government in Guidance of Local Science and Technology Development in Hubei Province (2020ZYYD040), the second batch of the Key Research and Development Project of Hubei Province (2020BAB073), the Outstanding Young and Middle-aged Scientific Innovation Team of Colleges and Universities of Hubei Province: "Biomass chemical technologies and materials" (Grant No. T201908), the Open Project of Key Laboratory of Green Chemical Process of Ministry of Education (GCP20200206), Hubei Key Laboratory of Novel Reactor and Green Chemical Technology (Wuhan Institute of Technology) (K202002).

Ethical StatementᄆThe manuscript was compliance with ethical approval and ethical standards. 
Author contributions: The manuscript was written through the contributions of all authors. All authors have given approval to the final version of the manuscript.

Conflicts of interest $\square$ The authors declare that they have no conflict of interest.

\section{References}

Apilux A, Siangproh W, Praphairaksit N, Chailapakul O (2012) Simple and rapid colorimetric detection of $\mathrm{Hg}$ (II) by a paper-based device using silver nanoplates Talanta 97:388-394

doi:10.1016/j.talanta.2012.04.050

Ding Y, Zhao J, Li B, Zhao X, Wang C, Guo M, Lin Y (2018) The CoOOH-TMB oxidative system for use in colorimetric and test strip based determination of ascorbic acid Microchimica Acta 185:131 doi:10.1007/s00604-018-2675-z

Esteban MA, Pei D (2012) Vitamin C improves the quality of somatic cell reprogramming Nature Genetics 44:366-367 doi:10.1038/ng.2222

Fang X, Wang J, Cui X, Zhang Y, Zhu R, Zhao H, Li Z (2019) Sensitive and facile colorimetric sensing strategy for ascorbic acid determination based on $\mathrm{CoOOH}$ nanoflakes-ABTs oxidative system Colloids and Surfaces A: Physicochemical and Engineering Aspects 575:66-74

doi:10.1016/j.colsurfa.2019.05.009

French AD (2013) Idealized powder diffraction patterns for cellulose polymorphs Cellulose 21:885-896 doi:10.1007/s10570-013-0030-4

French AD (2020) Increment in evolution of cellulose crystallinity analysis Cellulose 27:5445-5448 doi:10.1007/s10570-020-03172-z

Guadarrama-Fernandez L, Novell M, Blondeau P, Andrade FJ (2018) A disposable, simple, fast and lowcost paper-based biosensor and its application to the determination of glucose in commercial orange juices Food Chem 265:64-69 doi:10.1016/j.foodchem.2018.05.082

Han X-Y et al. (2019) Manganese(II)-doped zinc/germanium oxide nanoparticles as a viable fluorescent probe for visual and time-resolved fluorometric determination of ascorbic acid and its oxidase Microchimica Acta 186:466 doi:10.1007/s00604-019-3580-9

Ji D et al. (2018) A novel colorimetric strategy for sensitive and rapid sensing of ascorbic acid using cobalt oxyhydroxide nanoflakes and 3,3',5,5'-tetramethylbenzidine Sensors and Actuators B: Chemical 256:512-519 doi:10.1016/j.snb.2017.10.070

Jin R et al. (2019) Sensitive colorimetric sensor for point-of-care detection of acetylcholinesterase using cobalt oxyhydroxide nanoflakes J Mater Chem B 7:1230-1237 doi:10.1039/c8tb02987c 
Li N, Li Y, Han Y, Pan W, Zhang T, Tang B (2014) A Highly Selective and Instantaneous Nanoprobe for Detection and Imaging of Ascorbic Acid in Living Cells and in Vivo Analytical Chemistry 86:3924-3930 doi:10.1021/ac5000587

Li Z-M, Zhong X-L, Wen S-H, Zhang L, Liang R-P, Qiu J-D (2019) Colorimetric detection of methyltransferase activity based on the enhancement of $\mathrm{CoOOH}$ nanozyme activity by ssDNA Sensors and Actuators B: Chemical 281:1073-1079 doi:10.1016/j.snb.2018.11.085

Liu K, Yu P, Lin Y, Wang Y, Ohsaka T, Mao L (2013) Online Electrochemical Monitoring of Dynamic Change of Hippocampal Ascorbate: Toward a Platform for In Vivo Evaluation of Antioxidant Neuroprotective Efficiency against Cerebral Ischemia Injury Analytical Chemistry 85:9947-9954

Luo X, Lei X, Cai N, Xie X, Xue Y, Yu F (2016a) Removal of Heavy Metal lons from Water by Magnetic Cellulose-Based Beads with Embedded Chemically Modified Magnetite Nanoparticles and Activated Carbon ACS Sustainable Chemistry \& Engineering 4:3960-3969 doi:10.1021/acssuschemeng.6b00790

Luo X, Xia J, Jiang X, Yang M, Liu S (2019) Cellulose-Based Strips Designed Based on a Sensitive Enzyme Colorimetric Assay for the Low Concentration of Glucose Detection Anal Chem 91:15461-15468 doi:10.1021/acs.analchem.9b03180

Luo X et al. (2017) Improved Solid-Phase Synthesis of Phosphorylated Cellulose Microsphere Adsorbents for Highly Effective Pb2+ Removal from Water: Batch and Fixed-Bed Column Performance and Adsorption Mechanism ACS Sustainable Chemistry \& Engineering 5:5108-5117 doi:10.1021/acssuschemeng.7b00472

Luo X, Zhang H, Cao Z, Cai N, Xue Y, Yu F (2016b) A simple route to develop transparent doxorubicinloaded nanodiamonds/cellulose nanocomposite membranes as potential wound dressings Carbohydr Polym 143:231-238 doi:10.1016/j.carbpol.2016.01.076

Mahato K, Srivastava A, Chandra P (2017) Paper based diagnostics for personalized health care: Emerging technologies and commercial aspects Biosens Bioelectron 96:246-259 doi:10.1016/j.bios.2017.05.001

Nie Q, Cai Q, Xu H, Qiao Z, Li Z (2018) A facile colorimetric method for highly sensitive ascorbic acid detection by using $\mathrm{CoOOH}$ nanosheets Analytical Methods 10:2623-2628 doi:10.1039/C8AY00841H

Santos GPd, Corrêa CC, Kubota LT (2018) A simple, sensitive and reduced cost paper-based device with low quantity of chemicals for the early diagnosis of Plasmodium falciparum malaria using an enzymebased colorimetric assay Sensors and Actuators B: Chemical 255:2113-2120 doi:10.1016/j.snb.2017.09.005

Su L, Feng J, Zhou X, Ren C, Li H, Chen X (2012) Colorimetric detection of urine glucose based ZnFe2O4 marnetir nannnartirlec $\Delta$ nal Chem 84·5753-5758 doi:10.1021/ac300939z Loading [MathJax]/jax/output/CommonHTML/jax.js 
Tokura Y, Moriyama Y, Hiruta Y, Shiratori S (2019) Paper-Based Assay for Ascorbic Acid Based on the Formation of Ag Nanoparticles in Layer-by-Layer Multilayers ACS Applied Nano Materials 2:241-249 doi:10.1021/acsanm.8b01782

Tseng C-C, Yang R-J, Ju W-J, Fu L-M (2018) Microfluidic paper-based platform for whole blood creatinine detection Chemical Engineering Journal 348:117-124 doi:10.1016/j.cej.2018.04.191

Van Rie J, Thielemans W (2017) Cellulose-gold nanoparticle hybrid materials Nanoscale 9:8525-8554

Wang N, Sun J, Fan H, Ai S (2016) Anion-intercalated layered double hydroxides modified test strips for detection of heavy metal ions Talanta 148:301-307 doi:10.1016/j.talanta.2015.11.007

Wang YM, Liu JW, Jiang JH, Zhong W (2017) Cobalt oxyhydroxide nanoflakes with intrinsic peroxidase catalytic activity and their application to serum glucose detection Anal Bioanal Chem 409:4225-4232 doi:10.1007/s00216-017-0372-0

Wen S-H, Zhong X-L, Wu Y-D, Liang R-P, Zhang L, Qiu J-D (2019) Colorimetric Assay Conversion to Highly Sensitive Electrochemical Assay for Bimodal Detection of Arsenate Based on Cobalt Oxyhydroxide Nanozyme via Arsenate Absorption Analytical Chemistry 91:6487-6497 doi:10.1021/acs.analchem.8b05121

Xia J, Lei X, Lu Y, Liu S, Luo X (2020a) Coagulation mechanism of cellulose/metal nanohybrids through a simple one-step process and their interaction with $\mathrm{Cr}(\mathrm{VI})$ Int J Biol Macromol 142:404-411 doi:10.1016/j.ijbiomac.2019.09.112

Xia J, Zhang H, Yu F, Pei Y, Luo X (2020b) Superclear, Porous Cellulose Membranes with Chitosan-Coated Nanofibers for Visualized Cutaneous Wound Healing Dressing ACS Appl Mater Interfaces 12:2437024379 doi:10.1021/acsami.0c05604

Xie W, Tian M, Luo X, Jiang Y, He N, Liao X, Liu Y (2020) A dual-mode fluorescent and colorimetric immunoassay based on in situ ascorbic acid-induced signal generation from metal-organic frameworks Sensors and Actuators B: Chemical 302:127180 doi:10.1016/j.snb.2019.127180

Ye D et al. (2017) Deformation Drives Alignment of Nanofibers in Framework for Inducing Anisotropic Cellulose Hydrogels with High Toughness ACS Appl Mater Interfaces 9:43154-43162 doi:10.1021/acsami.7b14900

\section{Figures}



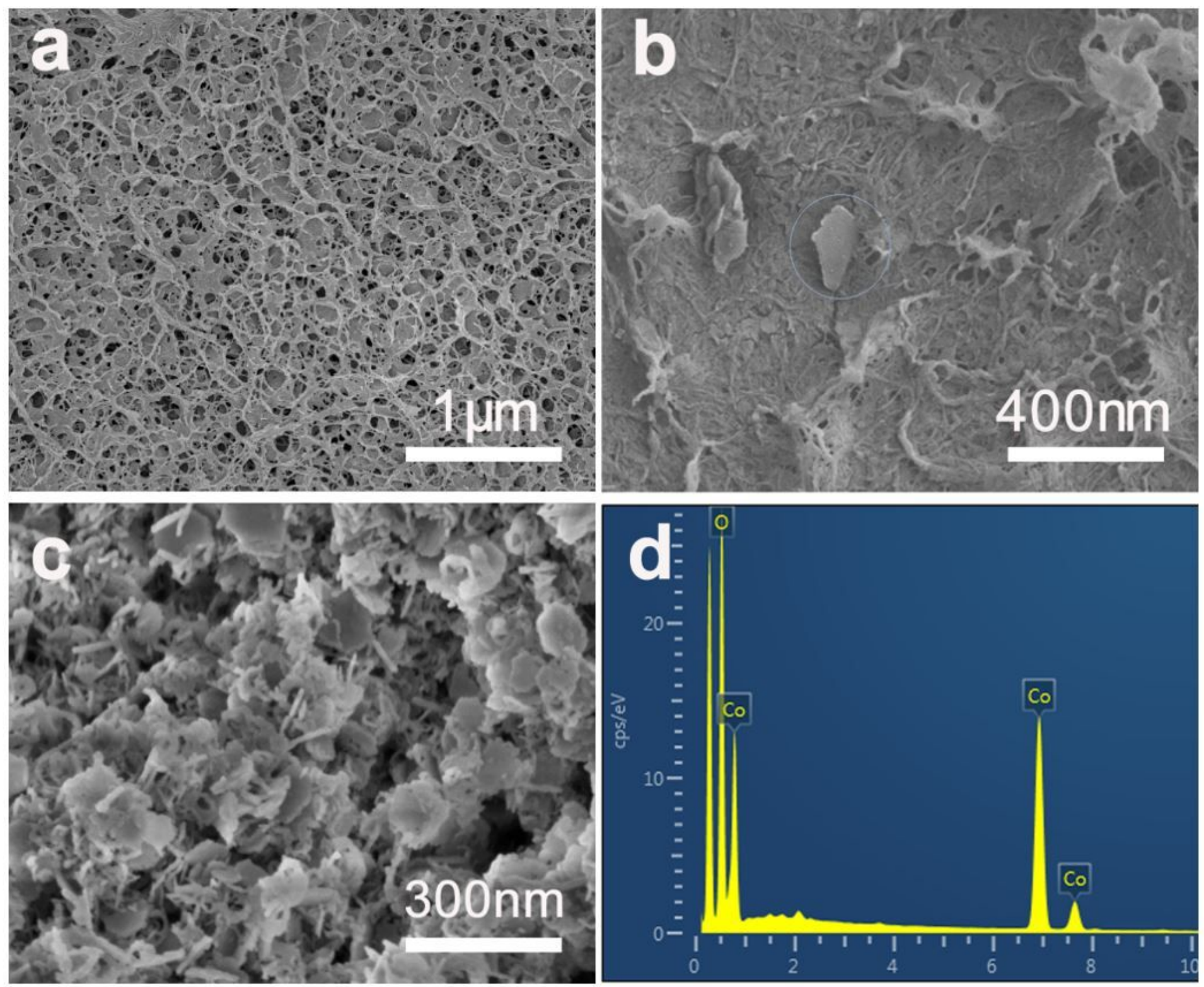

Figure 1

SEM images of (a) CM, (b) CCM, (c) $\mathrm{CoOOH}$ nanoflakes. (d) EDS pattern of pure $\mathrm{CoOOH}$ nanoflakes. 


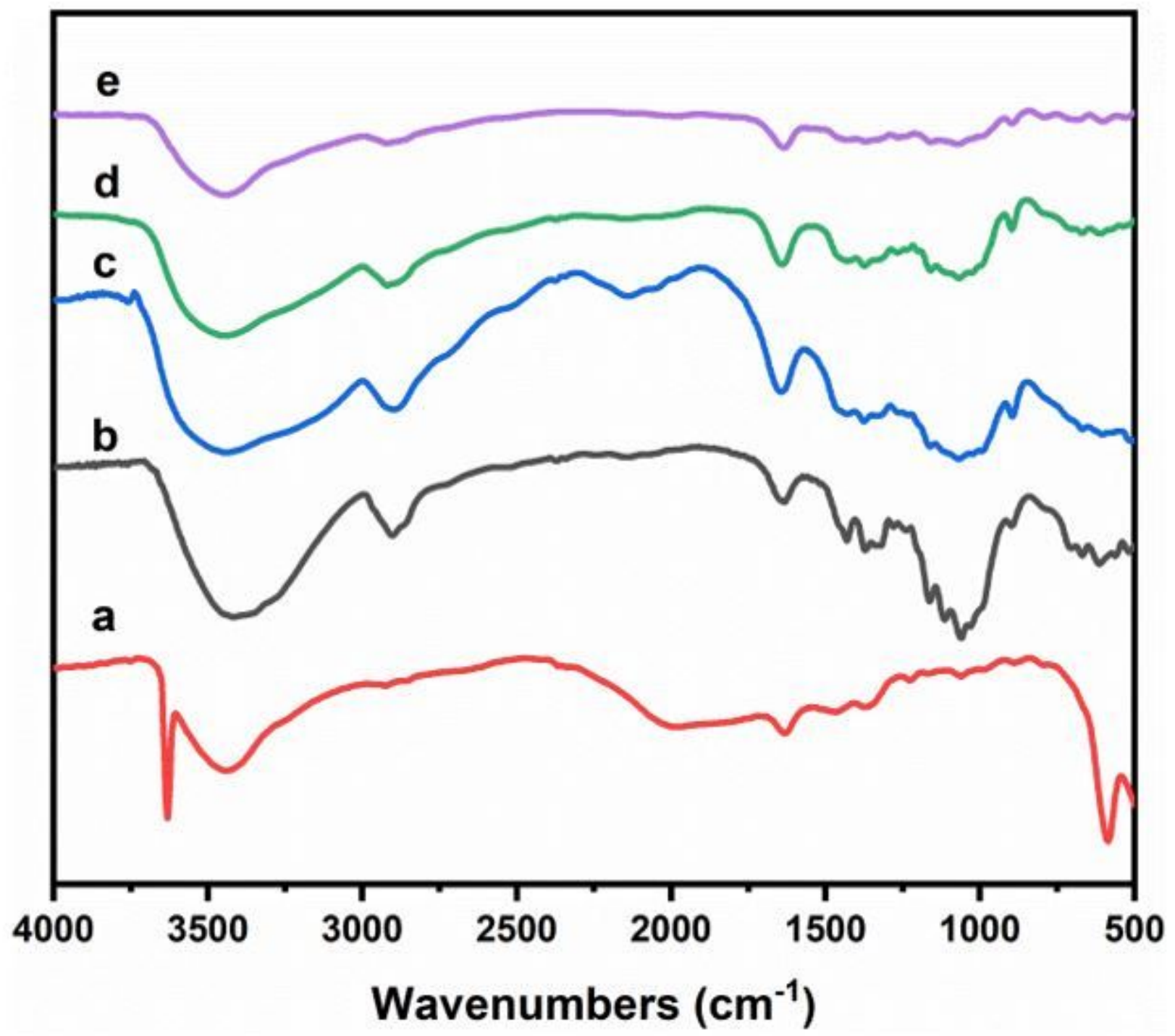

Figure 2

FT-IR spectra of $\mathrm{CoOOH}(\mathrm{a})$, cotton linter (b), CM (c), CCM (d) and CCM-AA (e). 


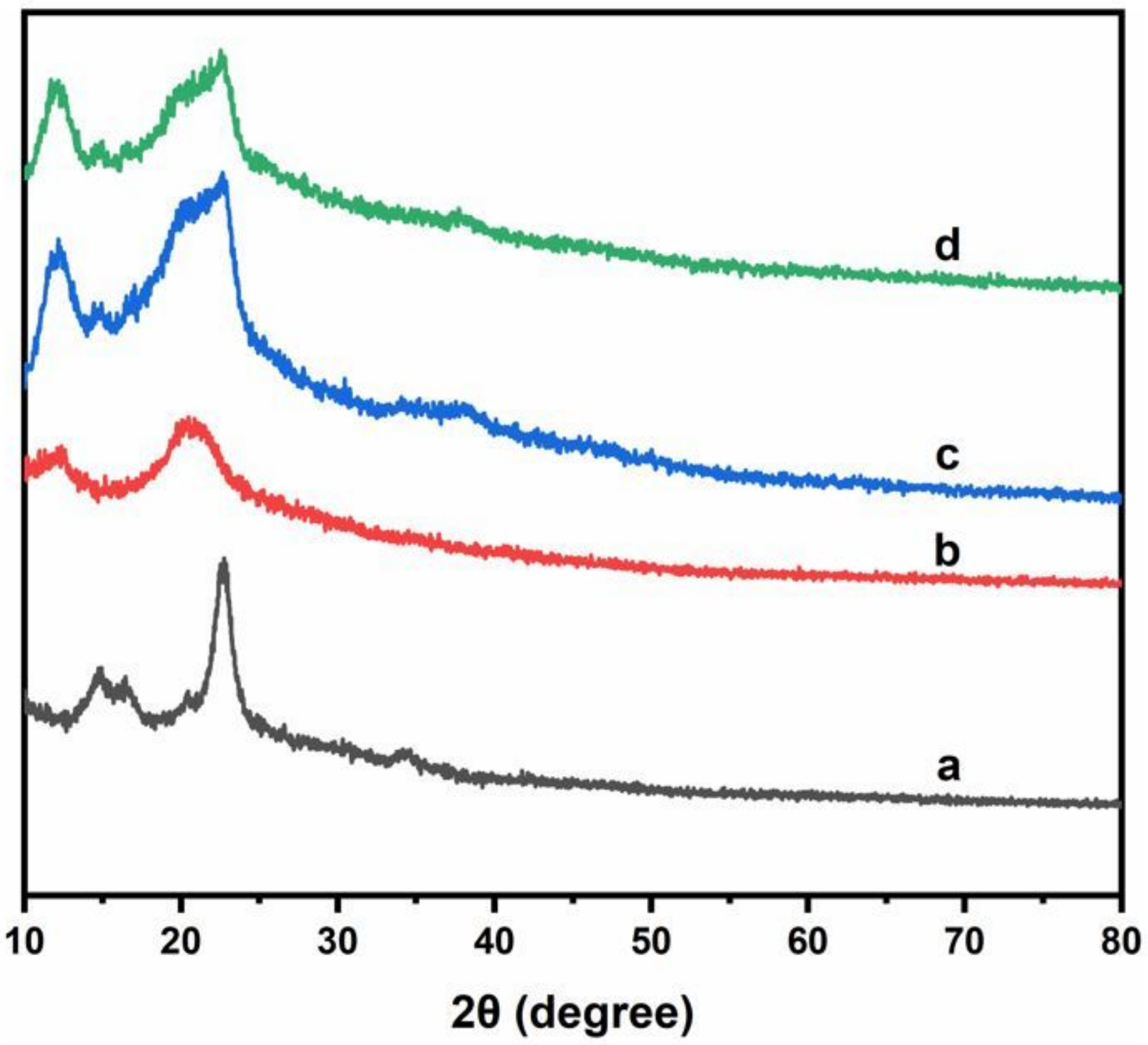

Figure 3

XRD patterns of cotton linter (a), CM (b), CCM (c) and CCM-AA (d). 

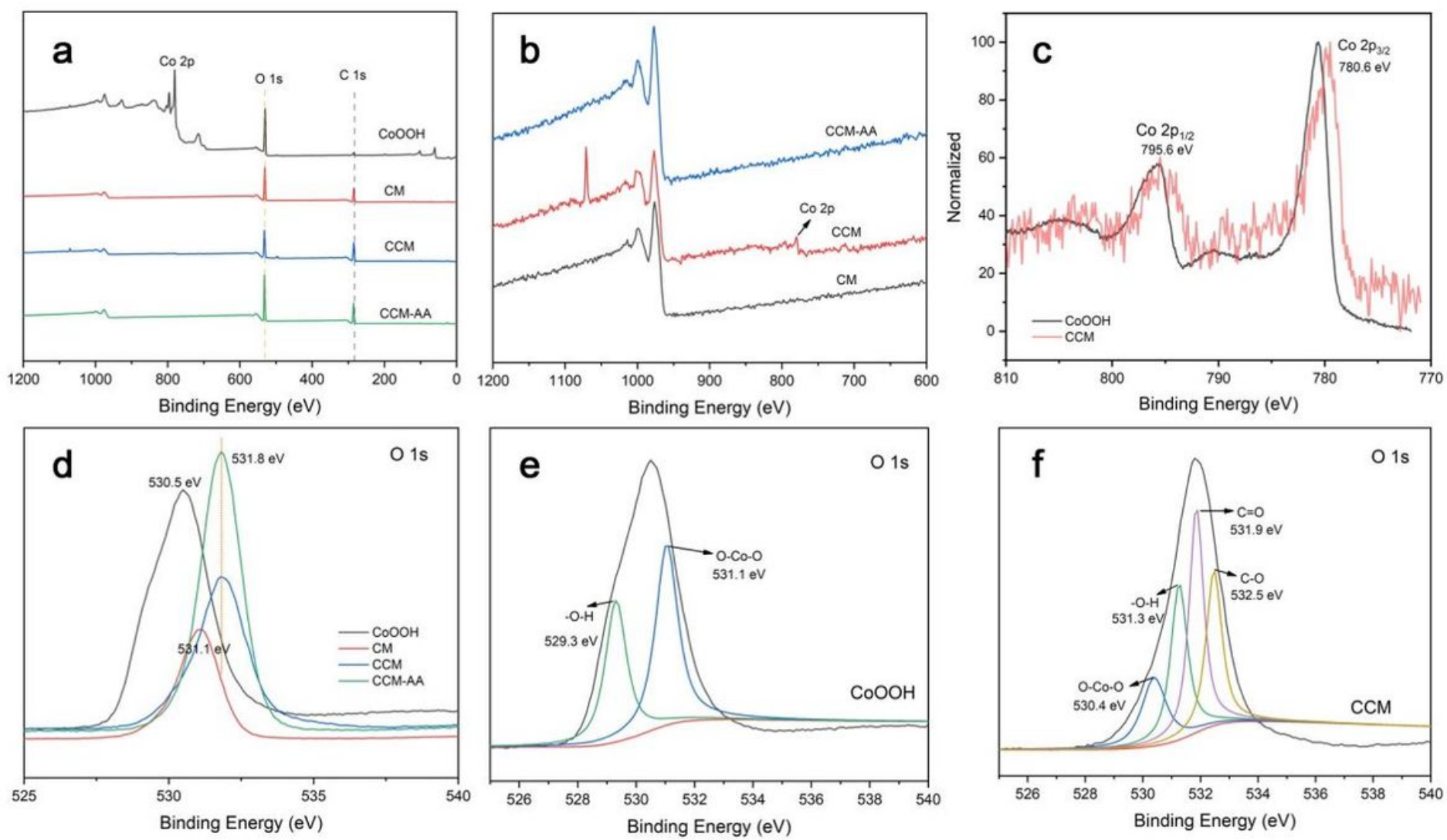

\section{Figure 4}

XPS spectra of $\mathrm{CoOOH}$ nanoflakes, CM, CCM, and CCM-AA: (a) Survey of $\mathrm{CoOOH}$ nanoflakes, CM, CCM, and CCM-AA; (b) Enlarge image of (a); (c) Co 2p of CoOOH nanoflakes and CCM; (d) 0 1s survey of $\mathrm{CoOOH}$ nanoflakes, CM, CCM and CCM-AA; (e) $\mathrm{O} 1 \mathrm{~s}$ of $\mathrm{CoOOH}$ nanoflakes; (f) $\mathrm{O} 1 \mathrm{~s}$ of CCM. 


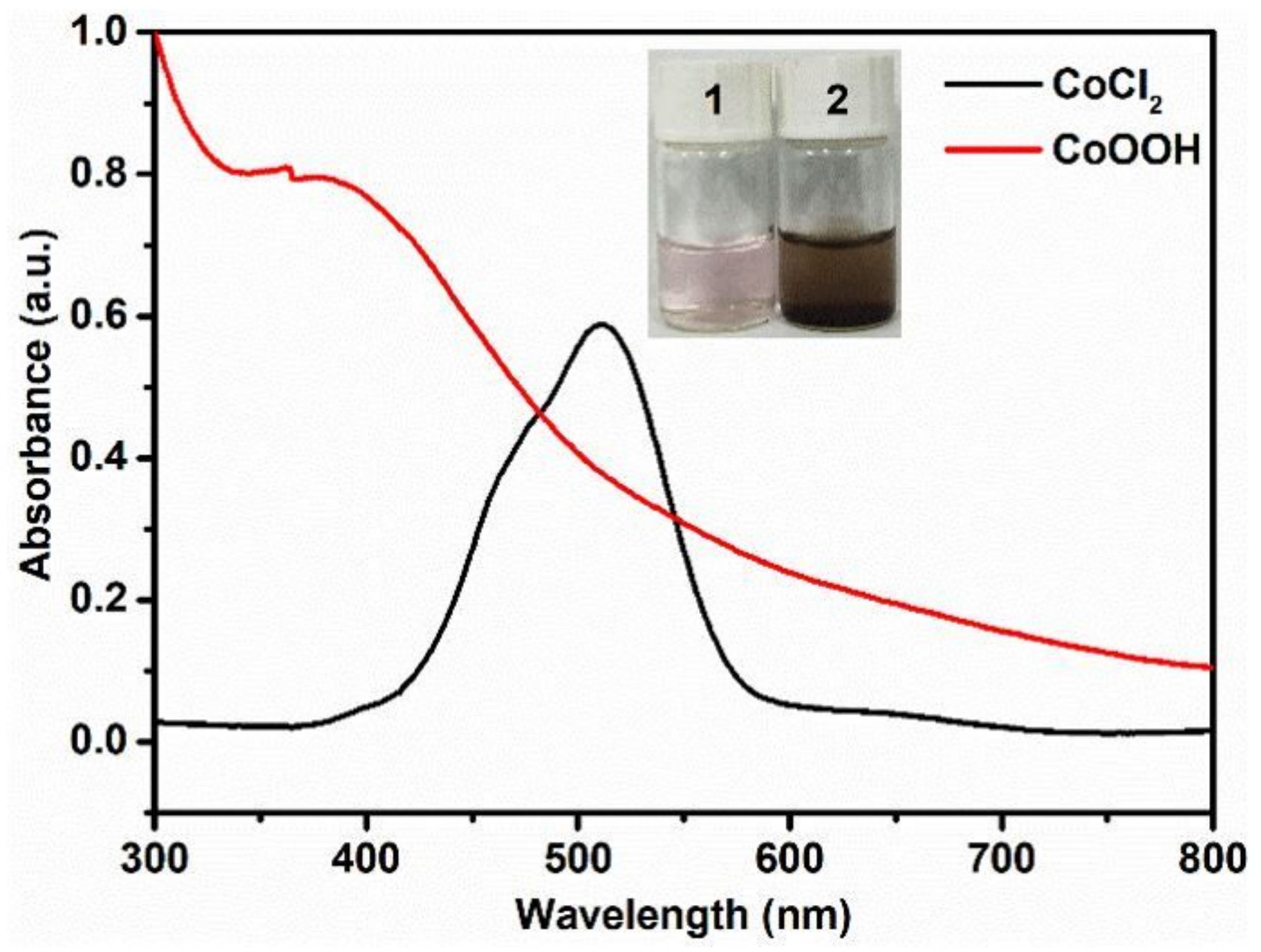

Figure 5

UV-vis absorption spectra of $\mathrm{CoOOH}$ nanoflakes (red curve) and $\mathrm{CoCl} 2$ solution (black curve), respectively. Inset: Digital images of $\mathrm{CoCl} 2$ solution (left) and $\mathrm{CoOOH}$ nanoflakes (right). 


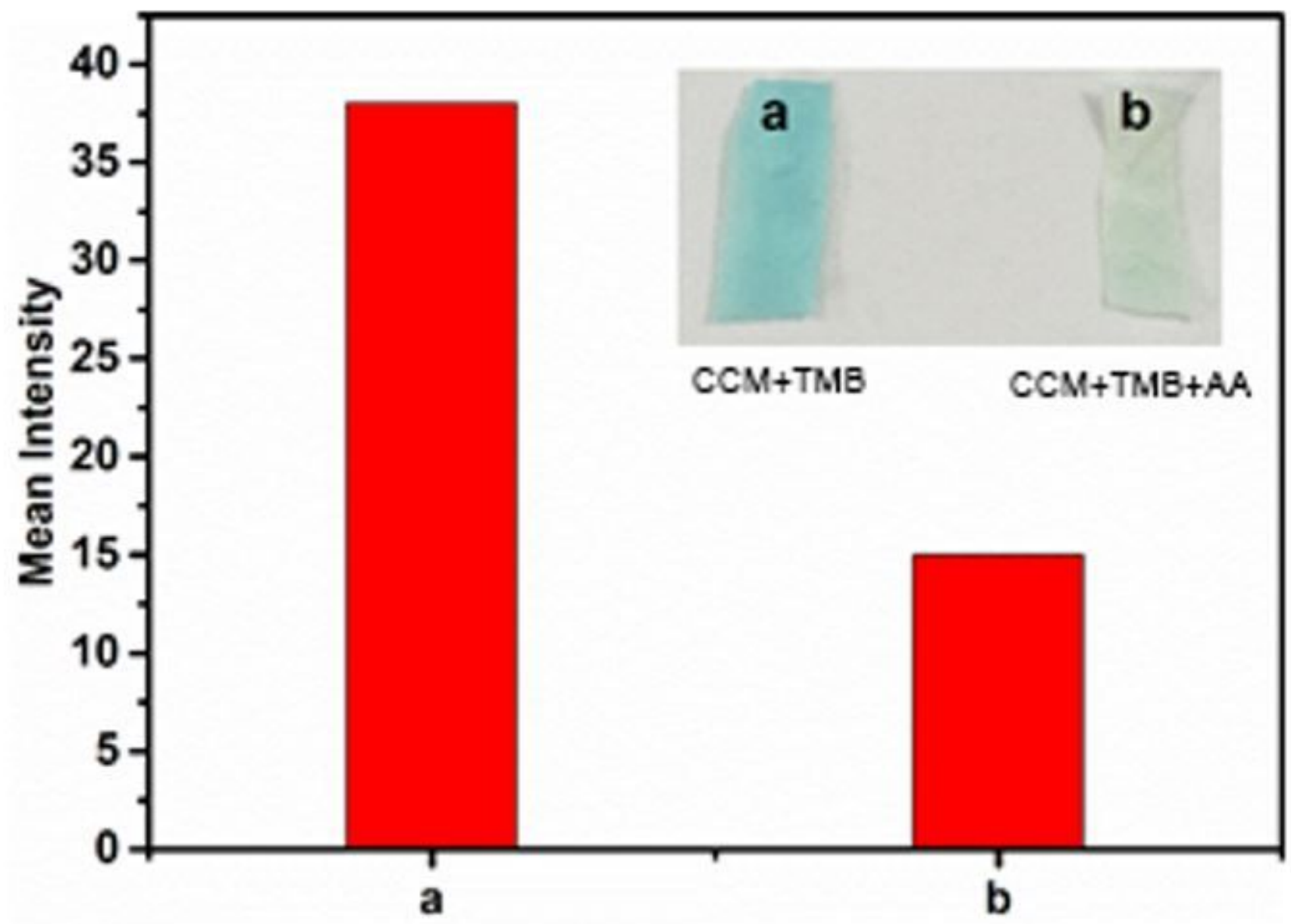

Figure 6

Colorimetric detection of AA by using the prepared CCM. Inset: the digital images of AA detection. 


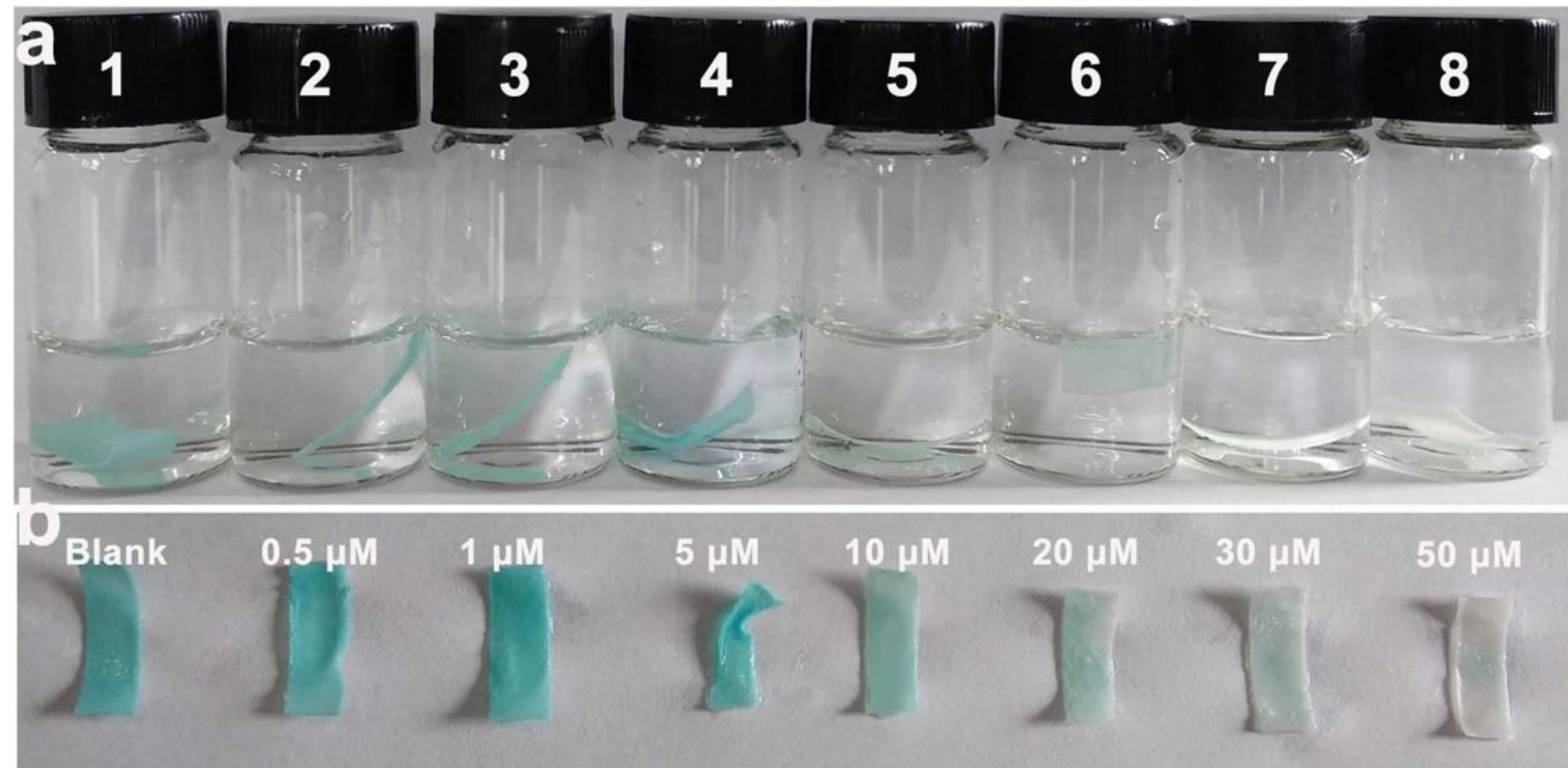

C
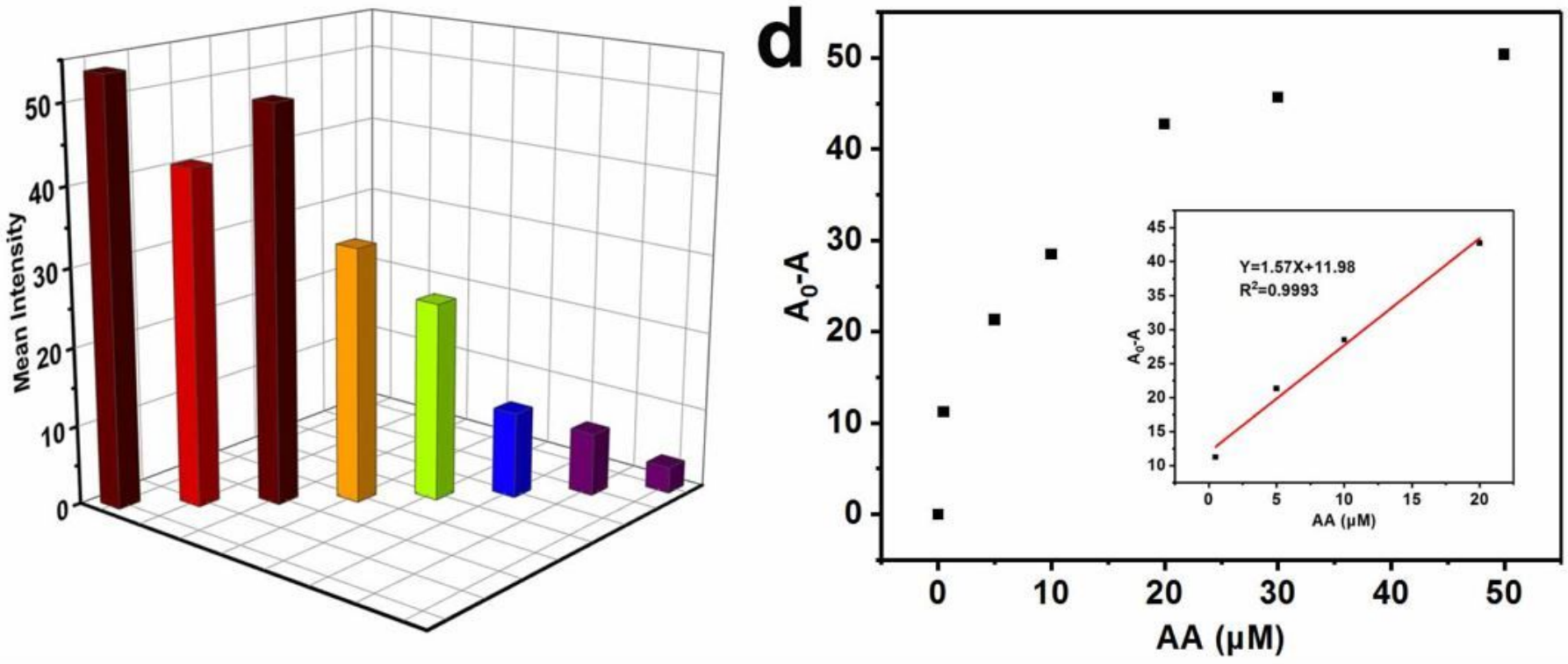

Figure 7

(a) The detection of $A A$ upon adding various concentrations of $A A$ : from 1 to 8 are $0 \mu \mathrm{M}, 0.5 \mu \mathrm{M}, 1 \mu \mathrm{M}, 5$ $\mu \mathrm{M}, 10 \mu \mathrm{M}, 20 \mu \mathrm{M}, 30 \mu \mathrm{M}$ and $50 \mu \mathrm{M}$. (b) Digital images of AA detection by using the CCM. (c) The mean intensity of CCM for visual detection of AA with different concentrations. (d) Linear relationship between (AO-A) and the concentrations of $A A(0-50 \mu M)$. Inset: linear relationship in the concentration range from $0.5 \mu \mathrm{M}$ to $20 \mu \mathrm{M}$ for $\mathrm{AA}$. $\mathrm{A}$ and $\mathrm{A} 0$ are the absorbance in the presence and absence of $\mathrm{AA}$, respectively. 

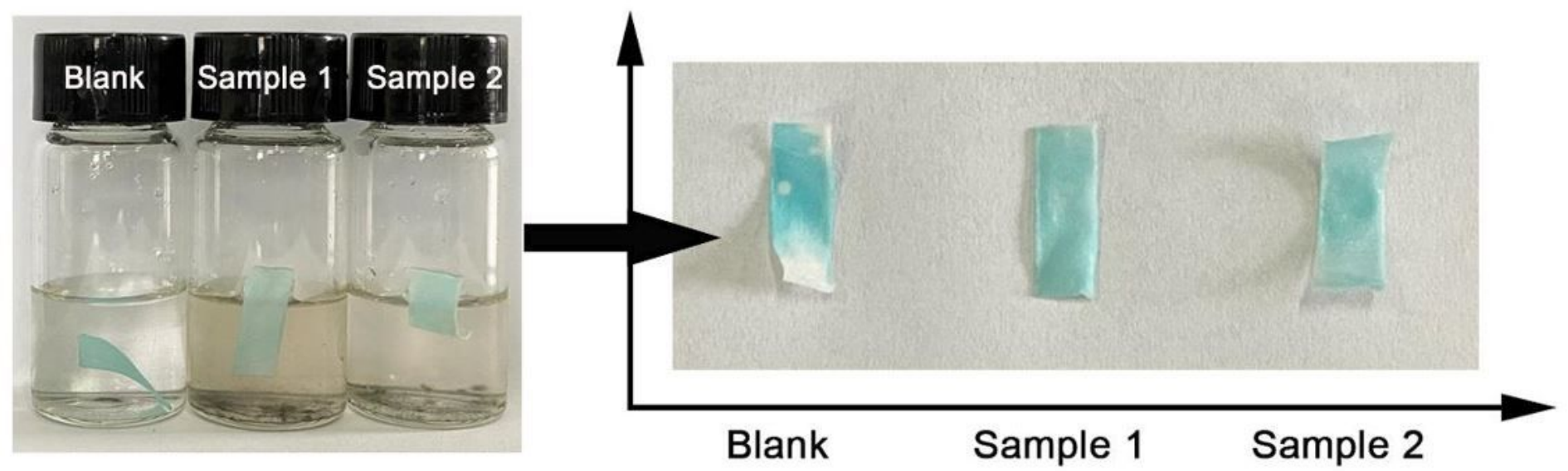

Figure 8

The detection of AA solution in real samples detection by using the CCM.
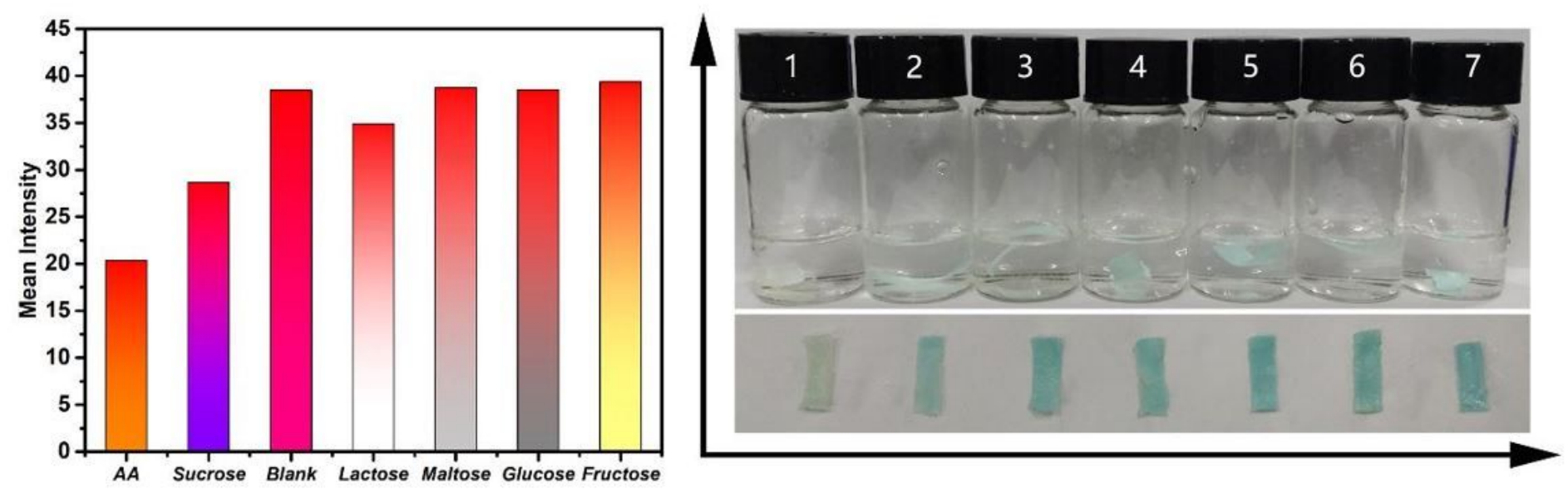

\section{Figure 9}

The visual detection of the other interferences by using the CCM. Left is the mean intensity of CCM for visual detection of the other interferences, and right is the digital images of CCM for visual detection of the other interferences. The concentration of $\mathrm{AA}$ is $50 \mu \mathrm{M}$, other interferences are $5 \mathrm{mM}$. 


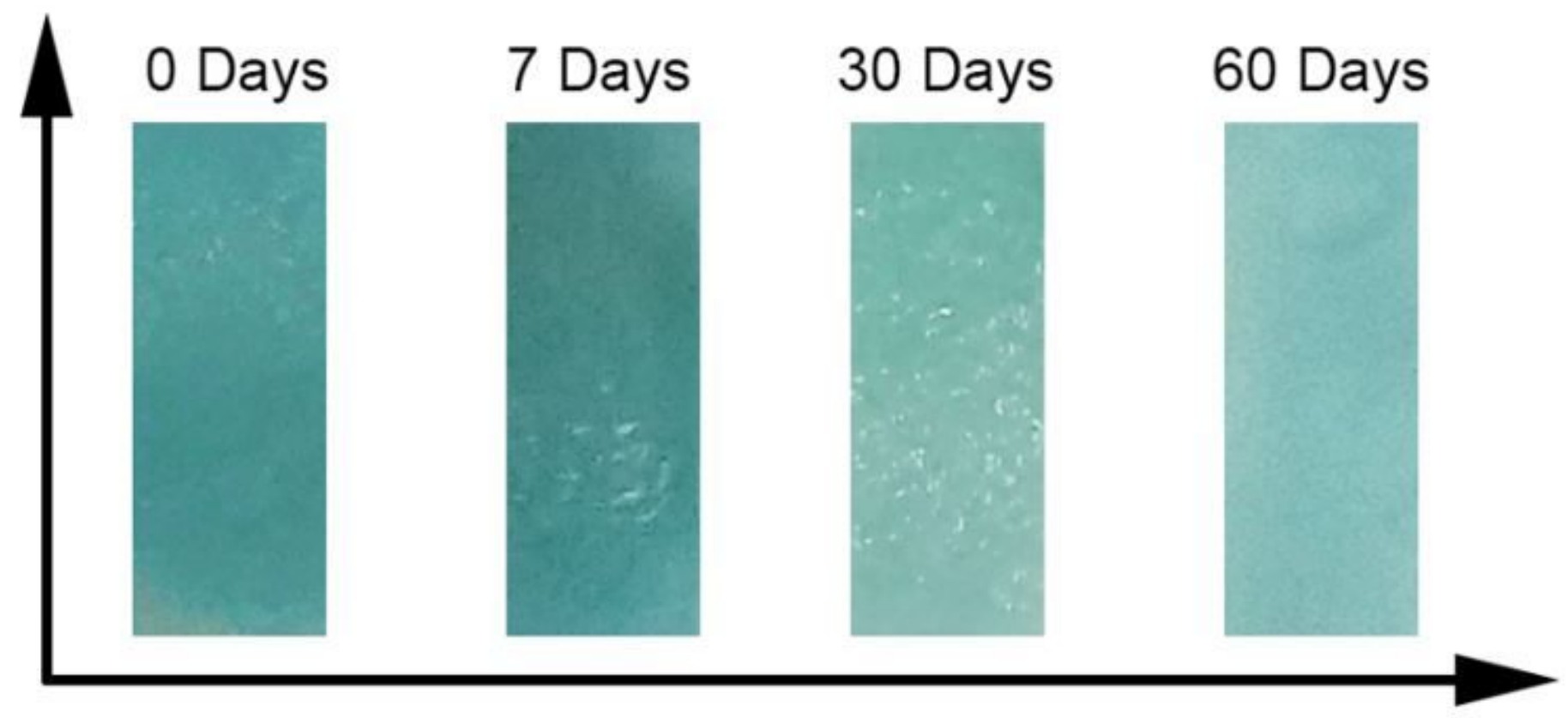

Figure 10

Colorimetric detection of AA by using the prepared CCM under different days.

\section{Supplementary Files}

This is a list of supplementary files associated with this preprint. Click to download.

- scheme1.jpg

- SupplementaryMaterial.docx 